\title{
Temporal Stochastic Channel Model for Absolute Polarization State and Polarization-Mode Dispersion
}

\author{
Cristian B. Czegledi ${ }^{\S}$, Magnus Karlsson ${ }^{\ddagger}$, Pontus Johannisson ${ }^{\dagger}$, and Erik Agrell ${ }^{\S}$ \\ ${ }^{\S}$ Department of Signals and Systems, ${ }^{\ddagger}$ Department of Microtechnology and Nanoscience, \\ Chalmers University of Technology, SE-412 96 Gothenburg, Sweden \\ ${ }^{\dagger}$ Sensor Systems, Acreo Swedish ICT AB, SE-41133 Gothenburg, Sweden \\ czegledi@chalmers.se
}

\begin{abstract}
We propose and validate a discrete-time channel model for the temporal drift of the absolute polarization state and polarization-mode dispersion for coherent fiber optic systems. The model can be used in simulations to test and develop DSP for coherent receivers.
\end{abstract}

OCIS codes: 060.0060 Fiber optics and optical communications, 060.1660 Coherent communications.

\section{Introduction}

Digital signal processing (DSP) assisted coherent receivers have proved to be very robust against typical transmission impairments, such as chromatic dispersion, rotations of the state of polarization (SOP), and polarization-mode dispersion (PMD). The design, test, and tuning of optimal DSP algorithms can be achieved by simulating fiber transmission using channel models, which should describe the behavior of the fiber as accurately as possible.

PMD arises in a fiber due to birefringence and its random orientation along the fiber. Environmental temperature variations and mechanical vibrations change the local birefringence of the fiber, leading to stochastic temporal PMD fluctuations. Birefringence arises in optical fibers due to their non-circular cores as a result of manufacturing imperfections and applied stress on the fiber during and after deployment. Birefringence varies the propagation speed of the electromagnetic wave through the fiber for different SOPs, leading to pulse broadening. Essentially, the $x$ and $y$ field components of an optical signal will have different transit times and the time difference between them is called differential group delay (DGD).

When modeling PMD, the fiber is viewed as a concatenation of several fiber segments with DGD, which are stable over long time periods, connected through time-varying short segments (assumed to have negligible DGD) that randomly scatter the SOP, leading to the stochastic temporal nature of PMD. In the limit of infinite number of concatenated segments, one obtains a Maxwellian distribution of the accumulated DGD, both in time and wavelength, a model well established in the classic PMD literature [1]. However, experiments suggest that a typical transmission link consists of only few such time-varying fiber segments, so called "hinges" [2]. These hinges could be amplifier sites, fiber segments exposed to temperature or mechanical variations in servicing huts, railroad bridges, etc. This model is illustrated in Fig. 1(left), where the fiber is modeled as a sequence of $N$ short segments corresponding to frequency-independent hinges $\mathbf{H}_{n}(k)$ that rotate the SOP and are concatenated with random birefringent sections $\mathbf{D}_{n}(f)$ corresponding to long buried fiber segments, which are frozen in time, at least over the time scales of interest. The DGD of each such long section is assumed to have a Maxwellian distribution with respect to frequency.

The statistical aspects of both the classical and hinge PMD models are well studied in the literature dating back to the late eighties. However, no PMD channel models that take into account the temporal drifts are available. Typical results were developed as differential equations modeling the PMD vector dependence on time and fiber length, and used to analyze and predict outage probabilities [3]; or fast SOP changes were induced in a dispersion-compensating module under laboratory conditions [4].

In this paper, we suggest a discrete-time channel model within the framework of the hinge model to simulate PMD that takes into account the temporal evolution of the SOP drift in each hinge. We derive the autocorrelation function (ACF) of the proposed model, both in time and frequency, and compare it to experimental data. We will see that simulations match experimental data very well, both in terms of ACF fitting and DGD visual agreement. The model can be used in simulations where stochastic temporal polarization fluctuation is an issue. For example, fiber-optic systems can be simulated, which can be useful to, e.g., characterize receivers performance that rely to a higher and higher degree on sophisticated DSP. Moreover, the existing PMD tracking algorithms can be optimally tuned or new, more powerful algorithms can be designed.

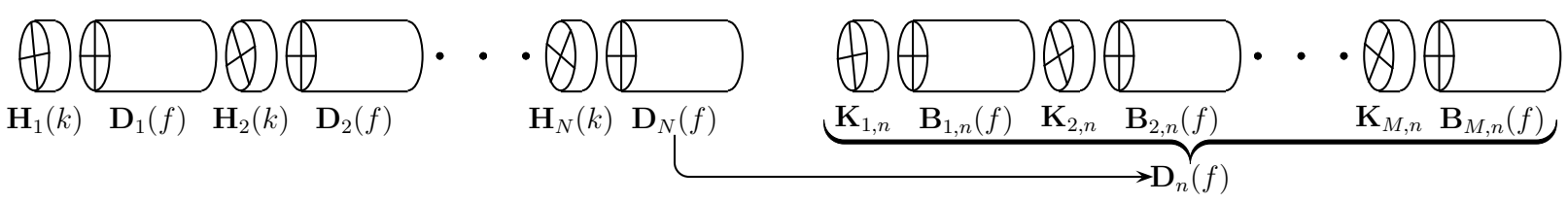

Fig. 1. Left: Model of a long fiber as a concatenation of static random birefringent sections $\mathbf{D}_{n}(f)$ coupled with time-varying hinges $\mathbf{H}_{n}(k)$. Right: Model of the random static sections $\mathbf{D}_{n}(f)$ as a concatenation of static birefringent sections $\mathbf{B}_{m, n}(f)$ coupled with static randomly oriented polarization rotators $\mathbf{K}_{m, n}$. 


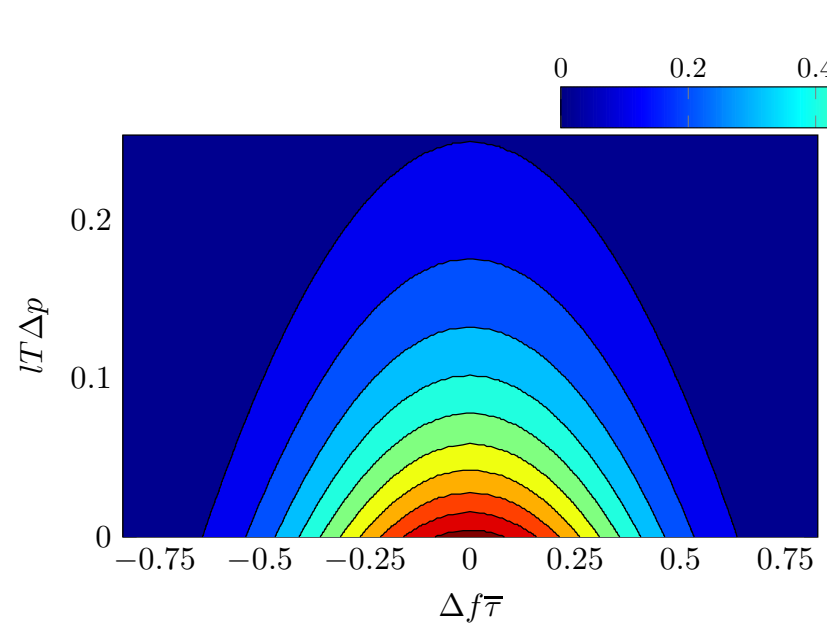

$\mathrm{ACF}$

$\begin{array}{llll}0.4 & 0.6 & 0.8 & 1\end{array}$

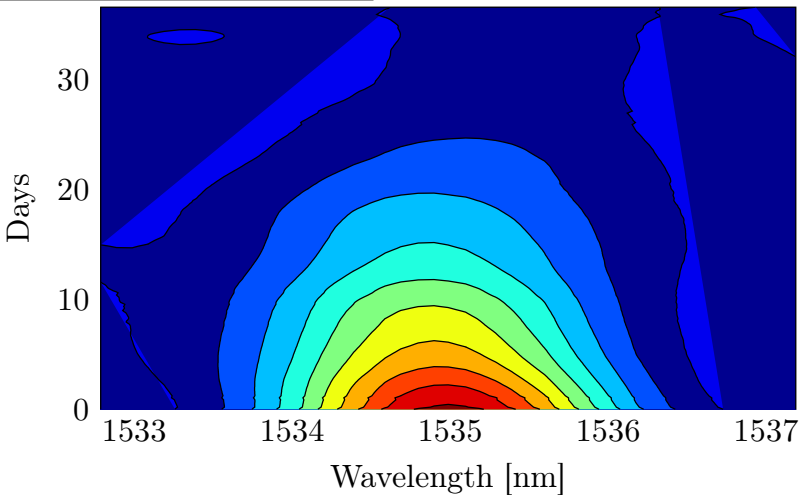

Fig. 2. The ACF is plotted as a function of (normalized) frequency on the horizontal axis and (normalized) time on the vertical axis of the proposed model (left) and compared with results obtained from experimental data (right). We can observe a very good (visual) agreement between the two, where the ACF from measurements has a fairly symmetric behavior and a similar relative decorrelation speed in time versus frequency.

\section{Proposed model}

The Jones matrix $\mathbf{T}(k, f)$ of the transmission link shown in Fig. 1 at time instance $k$ and frequency $f$ is given by

$$
\begin{aligned}
\mathbf{T}(k, f) & =\mathbf{D}_{N}(f) \mathbf{H}_{N}(k) \cdots \mathbf{D}_{2}(f) \mathbf{H}_{2}(k) \mathbf{D}_{1}(f) \mathbf{H}_{1}(k), \\
\mathbf{D}_{n}(f) & =\mathbf{B}_{M, n}(f) \mathbf{K}_{M, n} \cdots \mathbf{D}_{2, n}(f) \mathbf{K}_{2, n} \mathbf{B}_{1, n}(f) \mathbf{K}_{1, n},
\end{aligned}
$$

$$
\mathbf{B}_{m, n}(f)=\left(\begin{array}{cc}
e^{i \pi f \tau_{m, n}} & 0 \\
0 & e^{-i \pi f \tau_{m, n}}
\end{array}\right),
$$

for $n=1, \ldots, N$ and $m=1, \ldots, M$. In order to ensure that the DGD of $\mathbf{D}_{n}(f)$ is Maxwellian distributed and continuous with respect to frequency, we model $\mathbf{D}_{n}(f)$ in (2) as a sequence of $M$ independent and static polarization rotators $\mathbf{K}_{m, n}$ concatenated with static birefringent sections $\mathbf{B}_{m, n}(f)$ as shown in Fig. 1(right). For simplicity, we assume that the $N$ segments $\mathbf{D}_{n}(f)$ for $n=1, \ldots, N$ are composed by concatenating the same number of $M$ polarization rotators $\mathbf{K}_{m, n}$ and birefringent sections $\mathbf{B}_{m, n}(f)$ for $m=1, \ldots, M$. The polarization rotators $\mathbf{K}_{m, n}$ are static and randomly oriented such that they cover all the possible SOPs on the Poincare sphere uniformly. The birefringent sections $\mathbf{B}_{m, n}(f)$ are of equal mean DGD $\tau_{p}$, but random and independent for each section according to (3), where $\tau_{m, n} \sim \mathcal{N}\left(\tau_{p},\left(\tau_{p} / 5\right)^{2}\right)$ [5]. Choosing $\tau_{m, n}$ differently for each $\mathbf{B}_{m, n}(f)$ avoids spectral periodicity with period $\tau_{m, n} / 2$ of $\mathbf{D}_{n}(f)$, and consequently of $\mathbf{T}(k, f)$.

This model of $\mathbf{D}_{n}(f)$ in (2) has been used traditionally to emulate static PMD, where in the limit of $M \rightarrow \infty$ the DGD of each segment $\mathbf{D}_{n}(f)$ follows a Maxwellian distribution in frequency. However, for a finite $M$, the distribution is truncated and the truncation occurs at the sum of the absolute DGD values $\tau_{m, n}$ for $m=1, \ldots, M$ [6]. The fiber modeled by $\mathbf{T}(k, f)$ can be viewed as a concatenation of $N M \mathbf{B}_{m, n}(f)$ sections, thus the mean DGD of the fiber can be computed by the well-know expression $\bar{\tau}=\sqrt{8 N M / 3 \pi} \tau_{p}[7]$.

We model the hinges $\mathbf{H}_{n}(k)$ connecting the segments $\mathbf{D}_{n}(f)$ as randomly time-varying polarization rotators that scatter the SOP isotropically on the Poincaré sphere. The temporal drift of the hinges $\mathbf{H}_{n}(k)$ is emulated as a sequence of random Jones matrices [8]

$$
\mathbf{H}_{n}(k)=J\left(\dot{\boldsymbol{\alpha}}_{n}(k)\right) \mathbf{H}_{n}(k-1),
$$

where $J(\boldsymbol{\alpha})=\exp (-i \boldsymbol{\alpha} \cdot \overrightarrow{\boldsymbol{\sigma}})$ is a matrix function and $\overrightarrow{\boldsymbol{\sigma}}$ is a tensor of the three Pauli spin matrices [1]. The components of $\dot{\boldsymbol{\alpha}}_{n}(k)$ are random and drawn independently from a zero-mean Gaussian distribution at each time instance $k: \dot{\boldsymbol{\alpha}}_{n}(k) \sim$ $\mathcal{N}\left(\mathbf{0},(2 \pi \Delta p T / N) \mathbf{I}_{3}\right)$, where $T$ is the sampling period and $\Delta p$ is the polarization linewidth [8], quantifying the speed of the SOP drift. Each hinge $\mathbf{H}_{n}(k)$ at the initial time $k=0$ is a "uniform random matrix" such that it transforms an input vector to any output vector by a uniform probability, i.e., uniformly covering the Poincaré sphere. Such a matrix can be obtained by $J(\boldsymbol{\alpha}(0))$, where $\boldsymbol{\alpha}(0)=\theta \mathbf{a}$ is formed from the unit vector $\left(\cos \theta, a_{1} \sin \theta, a_{2} \sin \theta, a_{3} \sin \theta\right)^{\mathrm{T}}=\mathbf{g} /\|\mathbf{g}\|$ and $\mathbf{g} \sim \mathcal{N}\left(\mathbf{0}, \mathbf{I}_{4}\right)$. The same holds for the static polarization rotators, i.e., $\mathbf{K}_{m, n}=J(\boldsymbol{\alpha}(0))$, which independently and uniformly scatter the SOP on the Poincaré sphere. The ACF of the channel matrix $\mathbf{T}(k, f)$ separated by a time and frequency difference of $l T$ and $\Delta f$, respectively, in response to a constant input $\mathbf{u}$ can be expressed as

$$
\mathcal{A}_{\mathbf{T}}(l, \Delta f)=\mathbb{E}\left[(\mathbf{T}(k, f) \mathbf{u})^{\mathrm{H}} \mathbf{T}(k+l, f+\Delta f) \mathbf{u}\right] \approx\|\mathbf{u}\|^{2} \exp \left(-3 \pi|l| T \Delta p-\frac{3 \pi^{3}(|\Delta f| \bar{\tau})^{2}}{16}\right) .
$$

Fig. 2 compares the ACF of the proposed model (left) with results obtained from experimental data (right). The experimental data was obtained by measuring the Jones matrices of a $127 \mathrm{~km}$ long buried fiber from $1505 \mathrm{~nm}$ to 1565 


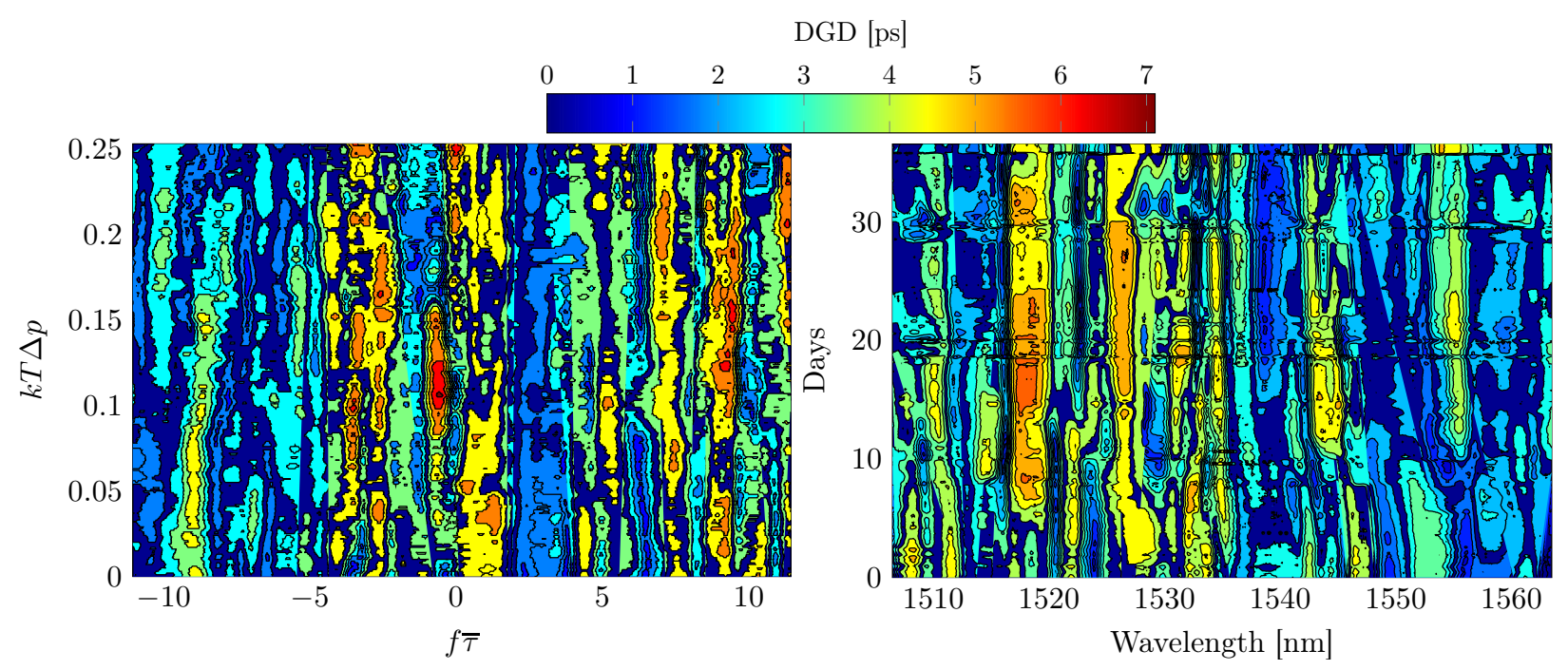

Fig. 3. DGD versus (normalized) frequency on the horizontal axis and (normalized) time on the vertical axis. Numerical DGD obtained from the model (left) yields a similar behavior as experimental data (right).

$\mathrm{nm}$ in steps of $0.1 \mathrm{~nm}$ for 36 days at every $\sim 2.2 \mathrm{~h}$. The fiber enters 12 hub stations, where a few tens of meters of fiber that were mechanically fixed are exposed to fluctuating air temperature, causing the temporal drift. The details of the measurement setup and postprocessing are published elsewhere [9]. The analytical ACF (5) can be fitted to measurements by setting $\bar{\tau}=2.95 \mathrm{ps}, T=2.2 \mathrm{~h}$ (both computed from the measurement data), $\Delta p=80 \mathrm{nHz}$, and $l=0, \ldots, 387$. The temporal drift of the fiber is caused to a large extent by the temperature fluctuations, which appear in the measurements as a slow drift on hourly and even daily cycles, thus explaining the very slow polarization drift.

In Fig. 3, we compare an example of the DGD obtained from the proposed model with experimental data [9]. We used the same parameters as above, i.e., $\bar{\tau}=2.95 \mathrm{ps}, \Delta p=80 \mathrm{nHz}$, and $T=2.2 \mathrm{~h}$, whereas the number of hinges was $N=12$ (same as the number of hubs), $M=1000$, and $k=1, \ldots, 388$. Comparing the two figures, we can notice a similar behavior, except some sudden changes occuring in the measured data around days 10, 18, 20, 30, and 35 . The implications of the hinge model [2] suggesting that not all spectral components are equally vulnerable to PMDinduced outages can also be noticed in Fig. 3, in both simulations and measurements. Most of the spectral components experience a low DGD for long time periods, resulting in a low outage probability, and few spectral components experience high DGD, making them more vulnerable to outages. During the measurement campaign, two fibers were measured and the DGD plot of the other fiber is published in [9], which also shows a similar behavior to the one shown here.

\section{Conclusions}

We proposed the first ever discrete-time channel model to simulate random temporal polarization fluctuations with PMD. The model is defined within the framework of the hinge model and has been successfully verified using experimental data measured on deployed fibers by comparing the ACF and DGD. The model can be used in simulations to emulate fiber transmission with time-varying PMD and to design, test, and tune DSP for coherent receivers, which was not possible with previous static models.

\section{References}

1. J. P. Gordon and H. Kogelnik, "PMD fundamentals: polarization mode dispersion in optical fibers," Proc. Natl. Acad. Sci. U.S.A. 97, 4541-4550 (2000).

2. M. Brodsky et al., "Polarization mode dispersion of installed fibers," J. Lightw. Technol. 24, 4584-4599 (2006).

3. C. Antonelli et al., "A model for temporal evolution of PMD," IEEE Photon. Technol. Lett. 20, 1012-1014 (2008).

4. G. Soliman et al., "Measurement and simulation of polarization transients in dispersion compensation modules," J. Opt. Soc. Am. A 27, 2532-2541 (2010).

5. C. H. Prola et al., "PMD emulators and signal distortion in 2.48-Gb/s IM-DD lightwave systems," IEEE Photon. Technol. Lett. 9, 842-844 (1997).

6. C. Antonelli and A. Mecozzi, "Theoretical characterization and system impact of the hinge model of PMD," J. Lightw. Technol. 24, 4064-4074 (2006).

7. C. Poole and D. Favin, "Polarization-mode dispersion measurements based on transmission spectra through a polarizer," J. Lightw. Technol. 12, 917-929 (1994).

8. C. B. Czegledi et al., "Polarization drift channel model for coherent fibre-optic systems," Sci. Rep. 6, 21217 (2016).

9. M. Karlsson et al., "Long-term measurement of PMD and polarization drift in installed fibers," J. Lightw. Technol. 18, 941-951 (2000). 